\title{
Innovative use of Single-stage Secondary Impression in treating Oral Rigidity due to Submucous Fibrosis
}

\author{
${ }^{1}$ Vishnu Manohar, ${ }^{2}$ YA Bindhoo, ${ }^{3}$ VR Thirumurthy, ${ }^{4}$ Anjana Kurien
}

\begin{abstract}
Restricted mouth opening of a patient presents a great challenge in prosthodontic rehabilitation, especially with complete dentures. Making an adequate border molded secondary impression is crucial in the success of such prostheses. Numerous techniques exist for making impressions in cases of microstomia. All of them involve sectional impressions and extraoral reorientation of the segments. Peripheral seal is compromised in these procedures. This article is laid out to explain how preserving border seal by using single-stage impression is by far the best technique found in the Prosthodontic literature to eliminate the cumbersome sectional impression method. A new method of preserving the seal by means of an impression recorded at a single stage is proposed.
\end{abstract}

Keywords: Border molding, Complete denture prosthodontics, Lego, Microstomia, Prosthodontics, Restricted mouth opening, Secondary impression, Sectional border molding, Sectional impression, Split tray.

How to cite this article: Manohar V, Bindhoo YA, Thirumurthy VR, Kurien A. Innovative use of Single-stage Secondary Impression in treating Oral Rigidity due to Submucous Fibrosis. Int J Prosthodont Restor Dent 2016;6(1):14-16.

Source of support: Nil

Conflict of interest: None

\section{BACKGROUND}

In India, a large population is addicted to areca nut chewing, which causes oral submucosal fibrosis. Oral submucous fibrosis is a condition that restricts opening of mouth, thereby restricting many of their oral functions. This condition was described by Pindborg ${ }^{1}$ as "an insidious, chronic disease affecting any part of the oral cavity and sometimes the pharynx, occasionally preceded by and/or associated with vesicle formation, it is always associated with juxta-epithelial inflammatory reaction followed by fibroelastic change of the lamina propria,

\footnotetext{
${ }^{1}$ Postgraduate Student, ${ }^{2}$ Reader, ${ }^{3}$ Professor and Head, ${ }^{4}$ Professor

${ }^{1-4}$ Department of Prosthodontics, Crown and Bridge including Implantology, Sri Ramakrishna Dental College and Hospital Coimbatore, Tamil Nadu, India,

Corresponding Author: Vishnu Manohar, Postgraduate Student, Department of Prosthodontics, Crown and Bridge including Implantology, Sri Ramakrishna Dental College and Hospital, Coimbatore, Tamil Nadu, India, Phone: +919952729312, e-mail: vichhukichhuvi@gmail.com
}

with epithelial atrophy leading to stiffness of the oral mucosa and causing trismus and inability to eat."

Oral submucous fibrosis results in marked rigidity and an eventual inability to open the mouth. This rigidity is an obstacle to prosthodontic procedures. A review of literature ${ }^{2}$ presents many such techniques that have been used such as reorienting elements following split custom trays, interlocking segments, and making the secondary impression in two stages and orienting it later extraorally. Various means include stainless steel posts inserted into tubings, ${ }^{3}$ die pins, ${ }^{4}$ orthodontic expansion screws, ${ }^{5}$ and Lego blocks. ${ }^{6,7}$ Complete peripheral seal cannot be evaluated, especially in the upper arch, as the impression is sectional. These methods are so painful to the patient, but the patient has to be called to the clinic multiple times to perfect the impression. It costs comfort and time for the patient.

In an attempt to overcome the shortcoming, a singlestage secondary impression is made following a regular sectional border molding. Posterior palatal seal and the complete border seal were achieved in the upper complete edentulous arch impression. Lego blocks were used for reorientation and two sets of locking mechanisms, one for intraoral reorientation and another for extraoral stabilization.

\section{CASE DESCRIPTION}

A 37-year-old female patient with difficulty in chewing food reported to the Department of Prosthodontics for replacement of teeth. History revealed that she had all upper and lower teeth extracted 2 years ago following dental caries. She complained of burning sensation when eating spicy and hot food and dryness of mouth.

Extraoral examination revealed that there was no facial asymmetry as well as no mandibular deviation upon opening and closing. Mouth rigidity presented as ability to open only $25 \mathrm{~mm}$. Intraoral examination revealed completely edentulous maxillary and mandibular arches, generalized blanching of the oral mucosa, and reduced salivation.

Preliminary impressions were made with irreversible hydrocolloid material (Tropicalgin, Zhermack, Italy). Her ability to open her mouth permitted the use of " 0 " size stock trays with some struggle. After the first appointment, the difficulty in border molding and 
achieving a good border seal was fathomed. The ridge was relatively flat and it was absolutely essential that a good border seal be achieved for fabrication of a retentive prosthesis.

Single-stage border molding would not have been possible for this case because of the restricted mouth opening. But the oral aperture was wide enough to insert and remove a complete tray. It was planned to fabricate a sectional impression tray for the case for peripheral tracing.

At the time of fabrication, after adapting an acrylic custom tray, Lego blocks (LEGO Systems, Enfield, Connecticut) were attached. Two pegged plates on either half with a reorienting block spanning across the midline were attached and designated as assembly "A." This helped in locking the segments intraorally during polymerization of the impression material.

Another extraoral locking feature was two lug-nutshaped pieces with internal grooves and a corresponding plastic rod with a " + " cross section being attached to the trays. This was designated as assembly "B." This served as a lock to stabilize the segments extraorally after polymerization of the impression material and before pouring in the master casts. Because of the " + "-shaped cross section, better stabilization and reorientation of the segments was achieved.

The assemblies " $\mathrm{A}$ " and " $\mathrm{B}$ " were attached to the custom tray by means of autopolymerizing acrylic on either side of midline with their respective keying elements locked. After the resin set, the keying elements of the assemblies were removed (Fig. 1). Both the upper and lower custom trays are sectioned in the middle. The trays are trimmed in such a way that, upon locking of the assembly "A", a gap of $1 \mathrm{~mm}$ will be left on either side of the midline. Peripheral tracing was completed with putty (Soft Putty, 3M ESPE, Korea) for the two sections of the custom tray for both upper and lower arches (Fig. 2).

Spacer wax was removed and no relief holes were provided, as the midline $1 \mathrm{~mm}$ gap acted as a region of relief in the midpalatal raphe region. This region also provides adequate bulk of light body elastomer to withstand the flexing load during retrieval of impression from the oral cavity. Tray adhesive (VPS Tray adhesive, 3M ESPE, Germany) was applied carefully over the impression surface and copiously over the midline region to facilitate adhesion of the impression material, with special care in the region that would flex.

Light body impression material (Express, 3M ESPE, USA) was loaded into both halves of the tray simultaneously and seated in the oral cavity. Assembly A was keyed with the block to lock the right and left segments spatially during polymerization of the elastomer (Fig. 3). After the impression was completely set, the key was removed first.

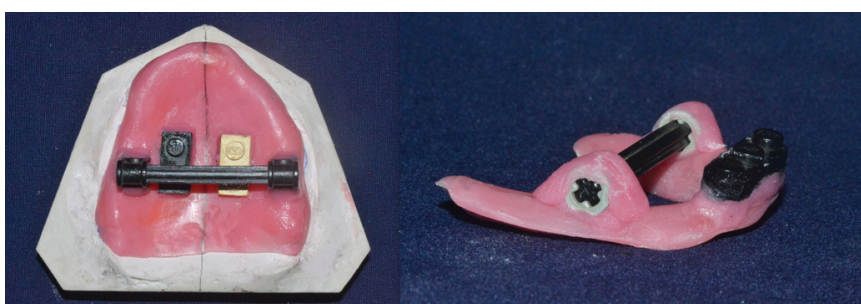

Fig. 1: Two pegged plates attached to the halves of the custom tray (for intraoral reorientation) and lug-nut-shaped pieces locked with "+" cross-section plastic rod element (for extraoral reorientation)

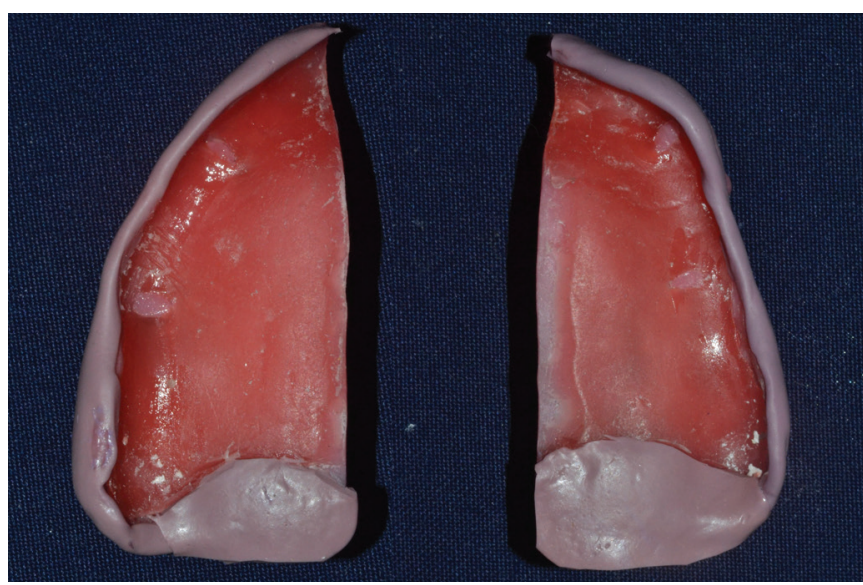

Fig. 2: Sectional border molding completed in two parts with putty impression material

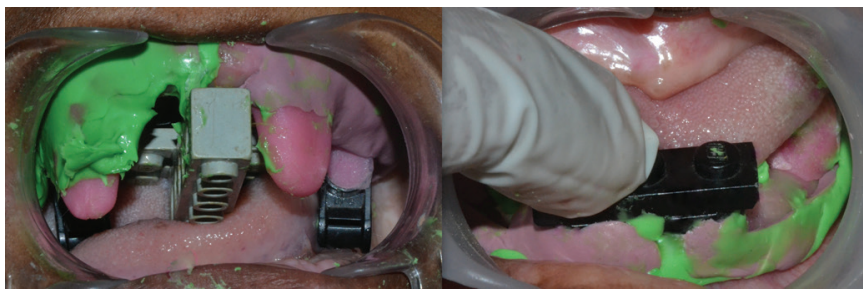

Fig. 3: Single-stage secondary impression with light-bodied elastomer with assembly "A" locked with intraoral keying blocks both for upper and lower arches

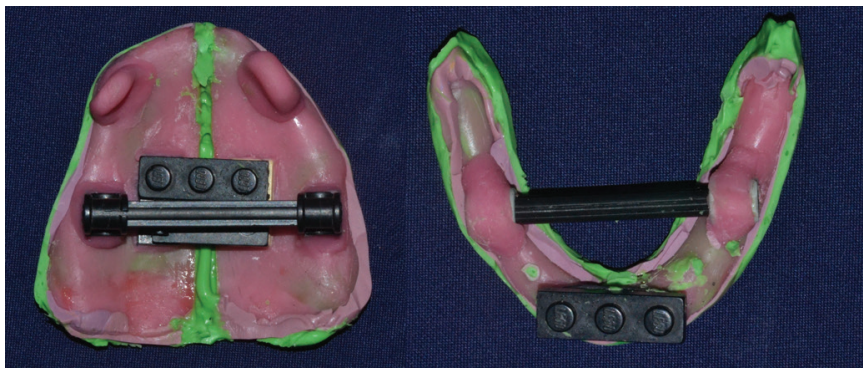

Fig. 4: Extraoral reorientation with the help of the assembly "B" locked with their respective "+" cross-section rods

The strip of light body impression material in-between the two halves of the custom tray served as a hinge, allowing leverage of the set impression (flexible at midline) and retrieval in a semi-collapsed form. Elastic recovery was permitted. Following elastic recovery, the assembly " $\mathrm{B}$ " was keyed with the rod (Fig. 4). This helped lock the two 


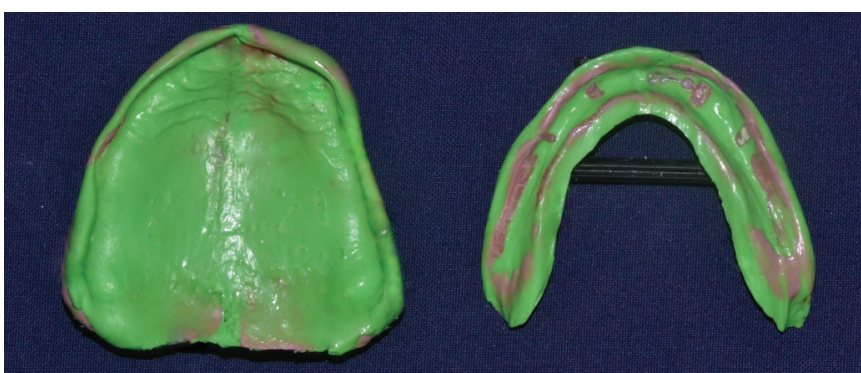

Fig. 5: Completed secondary impressions made by using the single-stage impression technique

halves of the tray, as designed at the time of fabricating. By following this protocol, a precise extraoral reorientation of the impression was achieved. The seal achieved intraorally was maintained and any cross arch tilt was nullified (Fig. 5). A single-stage impression procedure helped to achieve peripheral seal and ensure a retentive complete denture.

\section{DISCUSSION}

There are many alternative techniques available for making secondary impression in restricted mouth opening. ${ }^{2-7}$ But the main shortcoming of such sectional impression techniques is the difficulty in obtaining an adequate peripheral seal as the impression is made in two halves and the seal breaks in most cases. The protocol explained in this article proposes a method by which a single-stage impression can be made preserving the peripheral seal with the impression made as a whole. The elastic property of light-bodied impression material is utilized to create an area where the completed impression can be bent to remove it wholly. The use of two reorienting elements further improves accuracy by cross verification and elimination of any distortion as a result of the midline flexion. It further simplifies the procedure of obtaining casts by eliminating additional steps of pouring the cast in two segments and subsequent aligning of these parts.

\section{CONCLUSION}

Restricted mouth opening calls for alternative modalities of complete denture prosthodontics. The use of sectional border molding and impression techniques that are being practiced now has caused misalignment of dentures and discomfort to patients. Single-stage secondary impression promises accuracy and simplifies the orientation. It also preserves the border seal established, which is of paramount importance in the success of complete dentures.

\section{REFERENCES}

1. Pindborg JJ, Sirsat SM. Oral submucous fibrosis. Oral Surg Oral Medi Oral Pathol 1966 Dec;22(6):764-779.

2. Hegde C, Prasad K, Prasad A, Hegde R. Impression tray designs and techniques for complete dentures in cases of microstomia - a review. J Prosthodont Res 2012 Apr;56(2):142-146.

3. McCord JF, Tyson KW, Blair IS. A sectional complete denture for a patient with microstomia. J Prosthet Dent 1989 Jun;61(6):645-647.

4. Caculo SP, Aras MA, Chitre V. Fabrication of a custom sectional impression tray for a patient with oral submucous fibrosis. J Orofac Res 2013 Apr-Jun;3(2):140-143.

5. Mirfazaelian A. Use of orthodontic expansion screw in fabricating section custom trays. J Prosthet Dent 2000 Apr;83(4): 474-475.

6. Luebke RJ. Sectional impression tray for patients with constricted oral opening. J Prosthet Dent 1984 Jul;52(1):135-137.

7. Suzuki Y, Abe M, Hosoi T, Kurtz KS. Sectional collapsed denture for a partially edentulous patient with microstomia: a clinical report. J Prosthet Dent 2000 Sep;84(3):256-259. 